\title{
Erratum to: Invasive urothelial carcinoma with chordoid features may be an ominous sign predicting sarcomatoid change: a case report of a bladder carcinoma
}

\author{
Shogo Tajima ${ }^{1}$ Tomoyuki Suzuki ${ }^{1}$ Yukio Yamada $^{2} \cdot$ Daisuke Minagawa $^{2}$. \\ Yukio Homma ${ }^{2}$ Masashi Fukayama ${ }^{1}$
}

Published online: 19 August 2015

(C) The Japanese Society for Clinical Molecular Morphology 2015

\section{Erratum to: Med Mol Morphol}

DOI 10.1007/s00795-015-0098-z

The corresponding author of the article would like to remove the author's name "Teppei Morikawa" from the author group. The revised author group is as follows:

Shogo Tajima . Tomoyuki Suzuki . Yukio Yamada . Daisuke Minagawa · Yukio Homma · Masashi Fukayama

The online version of the original article can be found under doi:10.1007/s00795-015-0098-z.

Shogo Tajima

stajima-tky@umin.ac.jp

1 Departments of Pathology, Graduate School of Medicine,

The University of Tokyo, 7-3-1 Hongo, Bunkyo-ku,

Tokyo 113-0033, Japan

2 Department of Urology, Graduate School of Medicine,

The University of Tokyo, Tokyo, Japan 